\title{
Development of a Novel Double-Ring Deployable Mesh Antenna
}

\author{
Yan Xu, ${ }^{1}$ Fuling Guan, ${ }^{2}$ Xian Xu, ${ }^{2}$ Hongjian Wang, ${ }^{3}$ and Yao Zheng ${ }^{1}$ \\ ${ }^{1}$ School of Aeronautics and Astronautics, Zhejiang University, Hangzhou 310027, China \\ ${ }^{2}$ College of Civil Engineering and Architecture, Zhejiang University, Hangzhou 310058, China \\ ${ }^{3}$ Center for Space Science and Applied Research, CAS, Beijing 100190, China \\ Correspondence should be addressed to Xian Xu, xian_xu@zju.edu.cn
}

Received 5 July 2012; Accepted 19 October 2012

Academic Editor: Ahmed A. Kishk

Copyright (๑) 2012 Yan Xu et al. This is an open access article distributed under the Creative Commons Attribution License, which permits unrestricted use, distribution, and reproduction in any medium, provided the original work is properly cited.

This paper addresses a type of deployable mesh antenna consisting of the double-ring deployable truss edge frame and the cable net reflector. The structural design concept of the deployable antennas is presented. The deployable truss is designed and the geometric relationship of each strut length is formulated. Two types of radial truss elements are described and compared. The joint pattern and the active cables of the final design concept are determined. The pattern of the cable net is the three-orientation grid. Two connection schemes between the reflector and the deployable edge frame are investigated. The design parameters and the shape adjustment mechanism of this cable net are determined. The measurement test technologies of the antennas on the ground including test facilities, deployment test, and measurement and adjustment test are proposed. The antenna patterns are analyzed based on the real surfaces of the reflector obtained by the reflective surface accuracy measurement. The tests and analytic results indicated that the accuracy of the reflective surface is high and is suitable for low-frequency communication.

\section{Introduction}

The deployable mesh antenna consisting of the deployable edge frame and the spatially determined cable net has long been considered by space mission planners for the development of large-size spatial antennas. Many research and development efforts, covering innovative design concepts and analysis methods, were conducted to enhance the state of the-art of deployable antenna technology. The number of conference and journal papers published to report the results of these research efforts is very large $[1,2]$.

The recent research works focused on the cable net reflector including shape finding, prestress optimization and shape adjustment, and so forth. A new approach based on a force density strategy was presented to calculate a geodesic tension reflector that ensures both appropriate node position and uniform tension by Morterolle et al. [3]. The finite element model of cable net structure with active cables was established and active shape adjustment method was investigated by Wang et al. [4]. A surface distortions analysis method based on a first-order approximation to the aperture field was proposed for the hoop/column deployable mesh antennas [5]. The structure-electromagnetic analyses of a large deployable mesh antenna were presented, and the influences of structure deformations to the electromagnetic performances are concluded by Zheng et al. [6]. Some new design concepts of the deployable edge frames were presented and investigated for the deployable mesh antenna. Cherniavsky et al. described large space antennas based on the application of a closed polygonal pantograph truss, and, two means of maintaining the surface were discussed [7]. Takamatsu and Onoda proposed new concepts for three types of deployable large antenna structures and large solar concentrators [8]. Tsunoda et al. investigated the structural design and deployment test methods of a large deployable mesh reflector based on scissor-like element (SLE) [9]. A self-deployable structure with flexible joints was presented by Quirant et al. [10]. Most of these deployable edge frames are the single-ring truss. The structural stiffness of these singlering trusses is not enough for large-size spatial antennas and need to be reinforced [11].

This paper reports a recently conducted effort that developed an innovative design concept of mesh deployable antennas. The structural stiffness of the edge frames is improved, and the design concept is intended for large size mesh antennas. The deployable mechanics of the edge frames 


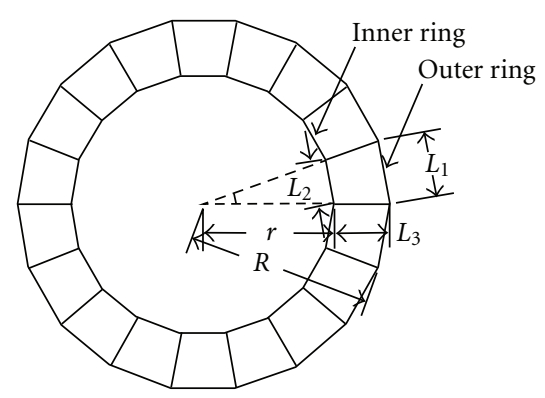

FIgURE 1: Top view of the deployable truss.

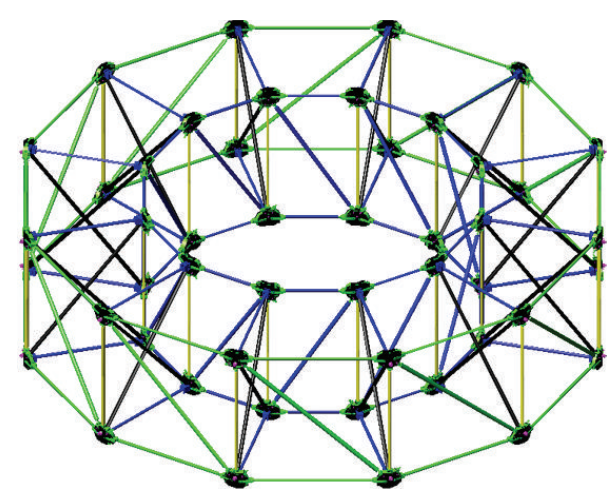

(a) The first truss concept

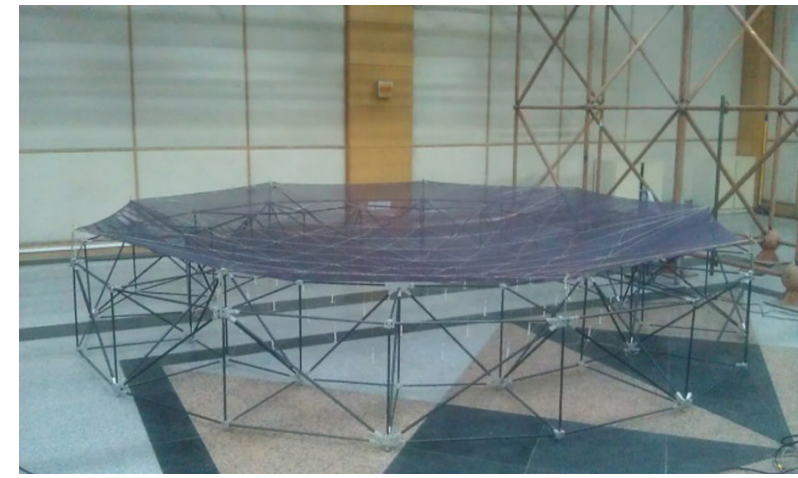

(b) The second truss concept

Figure 2: Two types of double-ring deployable truss concepts.

and the connection schemes of the cable net reflector to the edge frames need to be investigated carefully.

The remainder of this paper consists of four sections. Section 2 presents the structural design concept of the deployable mesh antennas. The double-ring deployable truss, the cable net, and the connection schemes are investigated. The measurement test technologies of the double-ring deployable mesh antennas on the ground are summarized in Section 3. The results of surface accuracy measurement test are used for pattern analysis in Section 4. Finally, Section 5 provides a few concluding remarks and suggestions for future work.

\section{Double-Ring Deployable Mesh Antennas}

For the mesh antennas, the most important component is the reflective surface. The reflective surface is stretched over the cable net and attached to the edge frames. The cable net consists of four kinds of cables: front, vertical, tie, and back cables. Front cables link the reflective surface nodes that are distributed uniformly on the parabolic surface. Therefore, the shape of reflective surface is determined by the location of the front cables. The vertical cables are for correcting the surface error which arises in the manufacturing process by adjusting the cable lengths.

It is difficult to satisfy the reflector precision requirement due to the flexibility of the cable net, especially for the space antenna with larger size. To reduce the surface error between the real reflector and the theoretical reflector, many qualifications are required for the structural design of the edge frames, such as the feasibility of deployment, the high stiffness, and the high packaging efficiency. A new design concept of deployable antenna is proposed and investigated, in which a double-ring deployable truss structure was employed as the edge frames.

2.1. Double-Ring Deployable Truss Structure. Two types of double-ring deployable truss are proposed, and the geometric equations of the truss structure are formulated in this section. The double-ring deployable truss is a symmetric structure and consists of $n$ spatial truss elements, as shown in Figure 1. There are two types of double-ring deployable truss concepts, as shown in Figure 2, in which the elements of the inner ring and the outer ring are the same and the radial truss elements are different.

There are two types of radial truss elements in the double-ring deployable trusses. The radial truss element in the first concept is a parallelogram mechanism, which is shown in Figure 3. The diagonal member can be folded and deployed by activator joints $\mathrm{F}$. The joints $\mathrm{F}$ consist of several torsion springs, as shown in Figure 3(c). The torsion springs are prestressed when the element is folded and rotate automatically after the binding is released. The diagonal member straightens (EF and FD are 180 degrees apart) and drives the entire element to deploy synchronously. To lock the element, the locking mechanism placed inside the activator joints is jumped by the tension spring and restricts the member. 


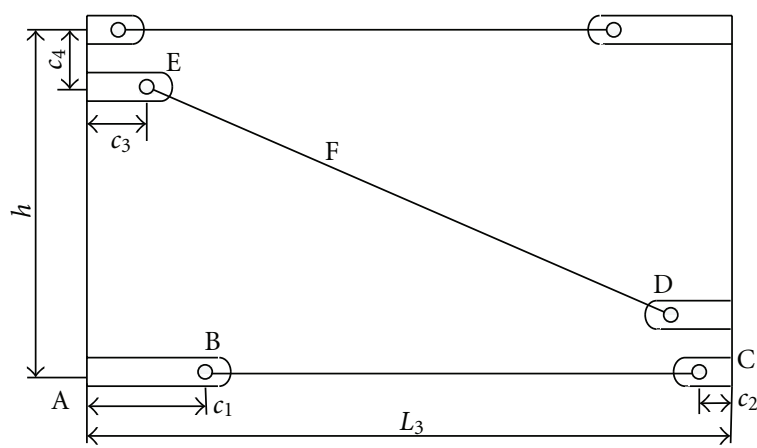

(a) Deployed configuration

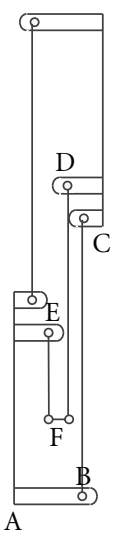

(b)

Folded

configu-

ration

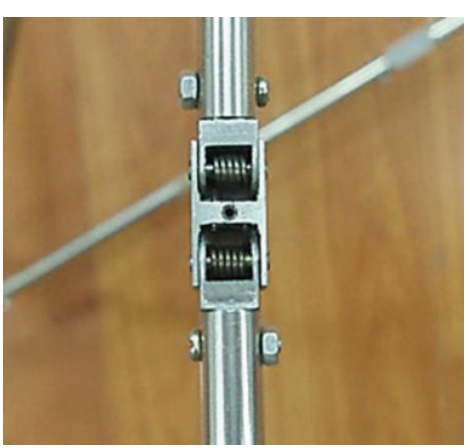

(c) Activator joint $\mathrm{F}$

Figure 3: Radial truss elements of the first truss concept.

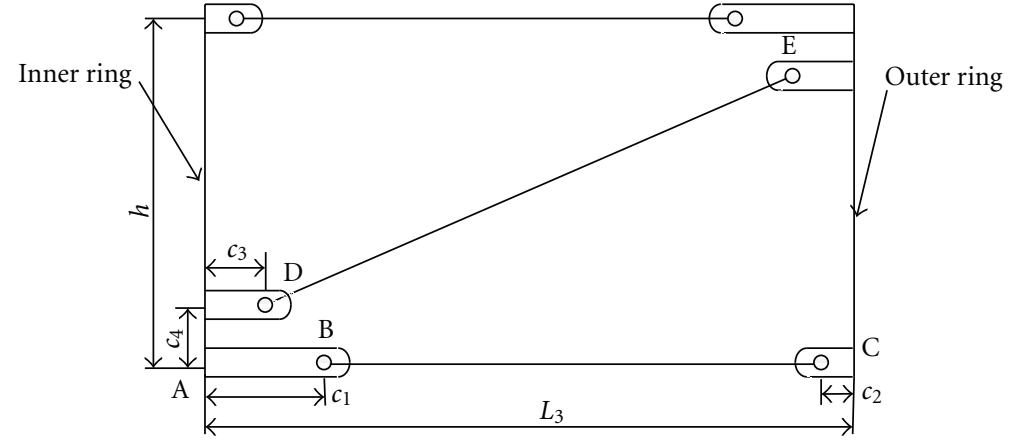

(a) Deployed configuration

FIgURE 4: Radial truss elements of the second truss concept.

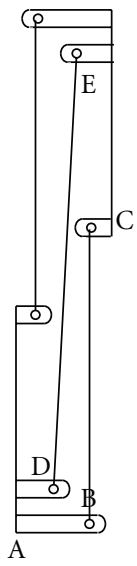

(b) Folded configuration

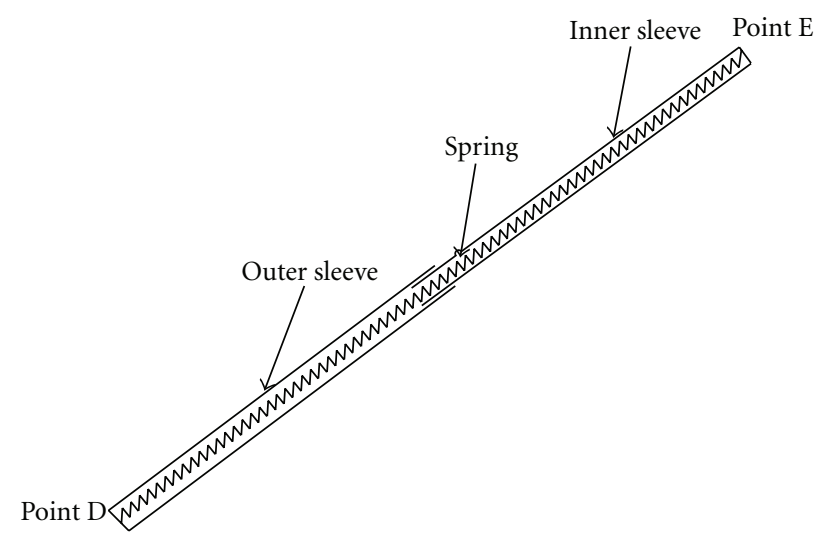

Figure 5: Activator struts DE. 


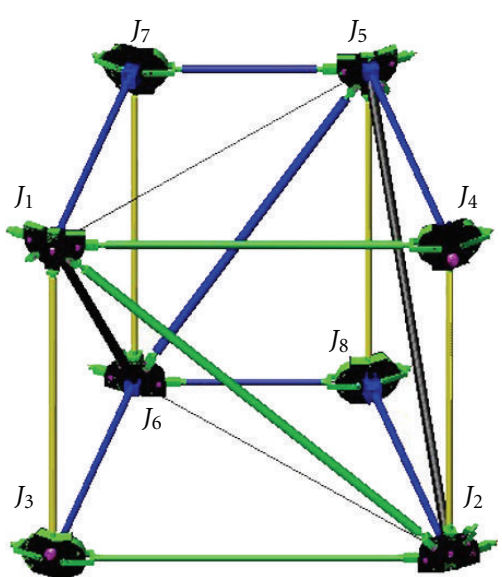

FIgURE 6: Spatial deployable element.

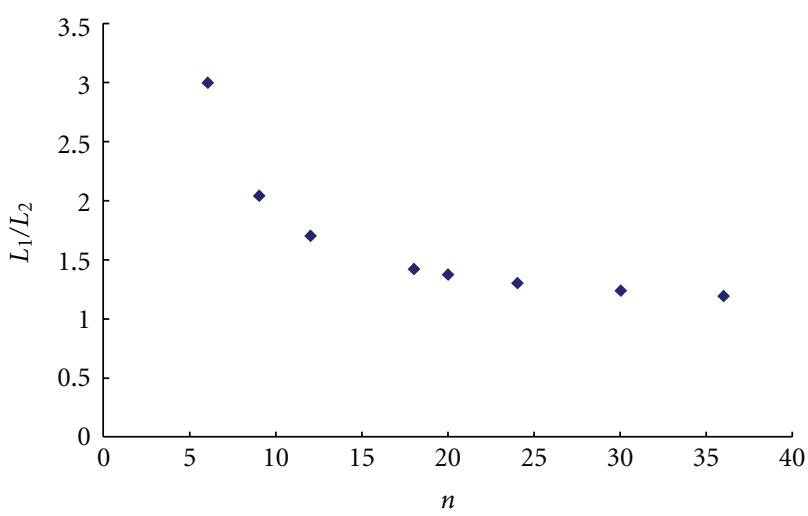

FIGURE 7: Variations of the length ratio with the side number.

The radial truss element of the second concept is also a parallelogram mechanism, which is shown in Figure 4. The element can be folded and deployed by the activator strut DE. There is a spring inside the strut DE, as shown in Figure 5. The spring is prestressed when the element is folded and deforms automatically after the binding of the whole truss in the folded configuration is released. The diagonal member shortens and drives the entire element to deploy synchronously. To lock the element, the locking mechanism placed inside the activator joints is jumped by the tension spring and restricts the member.

For the same section of the diagonal member, the stowed volume of the first concept is larger than that using the second one. The manufacture and assembly of the first concept is also more complex and difficult. Based on the comparison between these two types of double-ring deployable truss, the second concept was selected for the final demonstration model. The basic spatial deployable element of the demonstration model is a multiple-loop kinematic chain, shown in Figure 6. There are 8 joints in the element. The joints in the outer ring were numbered $J_{1}-J_{4}$, and the joints in the inner ring were numbered $J_{5}-J_{8}$. To analyse the length of each strut and the joint sizes, the geometric topology of the elements is investigated.
For the $N$-sided double-ring deployable truss, the circumference of the inner and outer rings are denoted by $r$ and $R$, respectively, as shown in Figure 1, and $h$ is the length of the vertical members. The length of the outer ring element, $L_{1}$, and the length of the inner ring element, $L_{2}$, can be expressed as

$$
L_{1}=2 R \sin \left(\frac{\pi}{n}\right), \quad L_{2}=2 r \sin \left(\frac{\pi}{n}\right) .
$$

The length of the radial truss element is

$$
L_{3}=R-r .
$$

The relationship between the circumradii of the inner and outer rings is

$$
R=\left(\frac{1+\sin (\pi / n)}{1-\sin (\pi / n)}\right) r
$$

This is the geometric equation that describes the double-ring deployable truss both deployed and folded, so the following relationships hold:

$$
\begin{aligned}
& \frac{L_{1}}{L_{2}}=\frac{L_{1}^{\prime}}{L_{2}^{\prime}}=\frac{1+\sin (\pi / n)}{1-\sin (\pi / n)}, \\
& \frac{L_{3}}{L_{2}}=\frac{L_{3}^{\prime}}{L_{2}^{\prime}}=\frac{1}{1-\sin (\pi / n)} .
\end{aligned}
$$

Referring to (4), the variations of the length ratio $L_{1} / L_{2}$ with the side number $n$ is depicted in Figure 7.

Then, the side number was determined as 18 , giving a length ratio $L_{1} / L_{2}=1.420$. The height of the deployable truss is $h=0.5 \mathrm{~m}$. The length of the outer ring element is $L_{1}=$ $0.8757 \mathrm{~m}$, and the length of the inner ring element is $L_{2}=$ $0.6167 \mathrm{~m}$.

As shown in Figure 8, there are two different types of joints in the element: one is denoted as a 7-strut joint and the other is denoted as a 4-strut joint. Based on the result of DOF evaluation analysis $[12,13]$, the DOF number of the deployable truss is 3 . Besides the activator struts shown in Figure 6, two active cables can be added in the diagonal members of the outer and inner ring truss element.

2.2. Cable Net Reflector. There are many kinds of cable network pattern for mesh antenna, such as radialized cable net, quasigeodesic cable net, and three-orientation grid cable net. After the comparison of the prestress distribution and the mechanical properties, the three-orientation grid cable net is determined for the deployable mesh antenna.

There are two schemes to connect the cable net reflector with the double-ring edge frame. The first one is shown in Figure 9(a), in which the cable net is attached in the inner ring truss. The second one is shown in Figure 9(b), in which the cable net is fixed in the outer ring truss. Because the height of the outer ring is the same as that of the inner ring, a short beam in the upper joint of the outer ring is need to avoid the reflective surface touching the inner ring truss in this scheme.

For the second scheme, there are some vertical cables placed between the outer ring and the inner ring truss. It is 


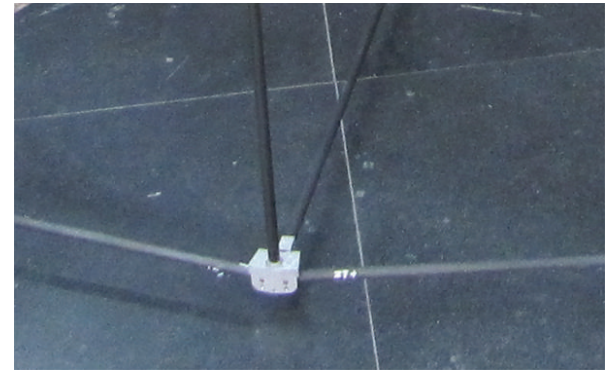

(a) 4-struts joint

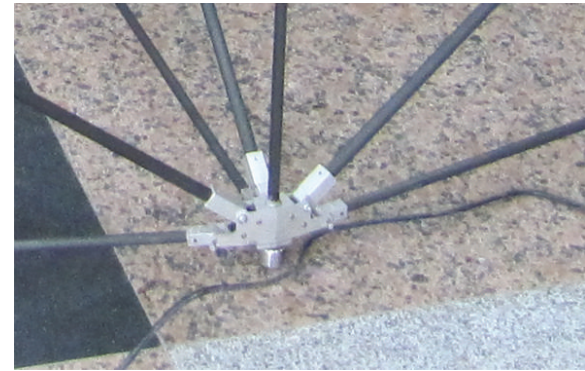

(b) 7-struts joint

Figure 8: Two types of truss element joints.

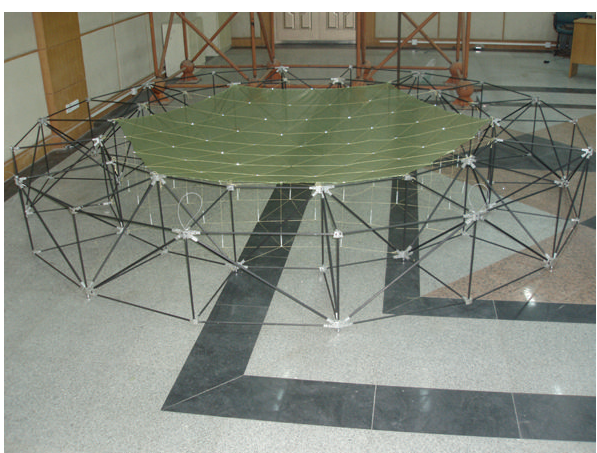

(a) The first scheme

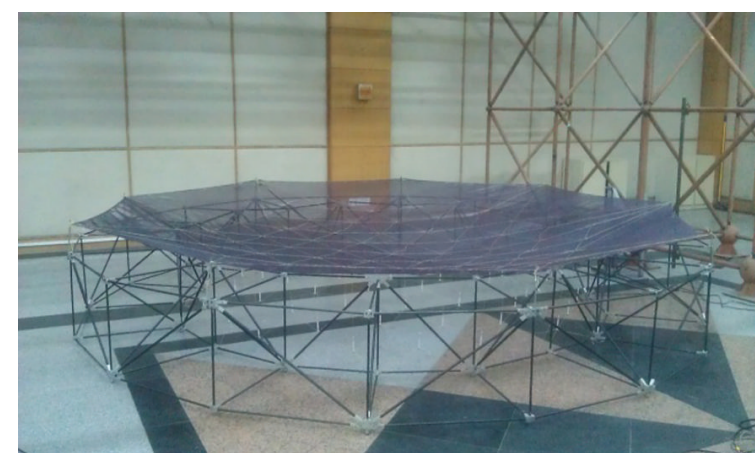

(b) The second scheme

Figure 9: Attachment schemes of the cable net to the truss.

difficult to avoid the physical interference between the cable net and the deployable truss during the deployment process. The height of the folded configuration of the second scheme is larger than that of the first scheme. The load path of the second scheme is less direct than the first approach. Though the size of the reflective surface of the second scheme is larger than that of the first scheme, the first scheme is used to design the cable net reflector.

To decrease the height of the mesh antenna, the focus length of the front cable net reflector and the back cable net surface are designed to be different values. The major design parameters of the reflective surface of the demonstration model include the following.

(i) The aperture of the reflective surface was $D=2.96 \mathrm{~m}$.

(ii) The ratio between the focus length and aperture of the front reflector was $F / D=1$.

(iii) The ratio between focus length and aperture of the back surface was $F / D=5$.

(iv) The mesh number of the radial cable is 4 .

(v) The mass of the entire model is $15.1 \mathrm{Kg}$.

(vi) The height of the folded configuration is $1.23 \mathrm{~m}$ and the diameter of that is $0.52 \mathrm{~m}$.

The coordinate of each joint and the geometric topology of each cable are determined by the equation of the surfaces and the three-orientation grid pattern. The geometrical model of the cable net reflector is shown in Figure 10.
Kevlar cord, $0.7 \mathrm{~mm}$ in diameter, was used for the cable net. An inverse iteration algorithm based on the finite element analysis was used to determine the pretension force distribution of the cable nets. A lightweight adjustment mechanism was designed to adjust the prestress of the vertical adjustable cables, as shown in Figure 11. The adjustment mechanisms are placed near the back cable net surface to make the adjustment easier.

\section{Mechanical Measurement Tests}

The purpose of this section is to sum up the measurement test technologies of the antennas on the ground. There are two important tests for the deployable mesh antenna: deployment test and surface accuracy measurement test. It is extremely difficult to evaluate the deployment characteristics of a large complete deployable structure on the ground. The gravity force affects the deployment process of the deployable mesh antenna heavily. The test facilities available to simulate space environments are designed for the deployment test. One type of these facilities is the suspend system, including the support frames, the plane orbit, the suspend cables, and the fix end mast, as shown in Figure 12. The deployment trace of each joint and strut in the antenna are obtained from the numerical simulation by the multibody dynamic theory. And these eighteen plane orbits are assembled under the support frames according to the deployment trace of the middle joint of the upper struts in the outer ring truss. 


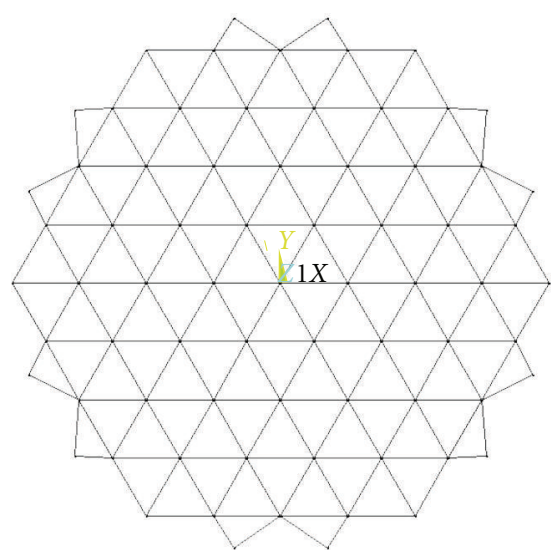

(a)

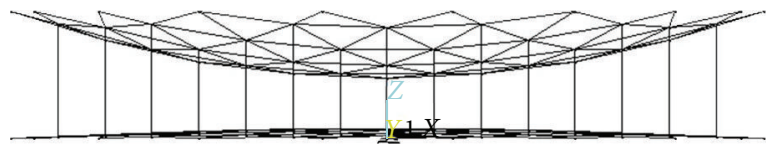

(b)

Figure 10: Geometrical model of the cable net.

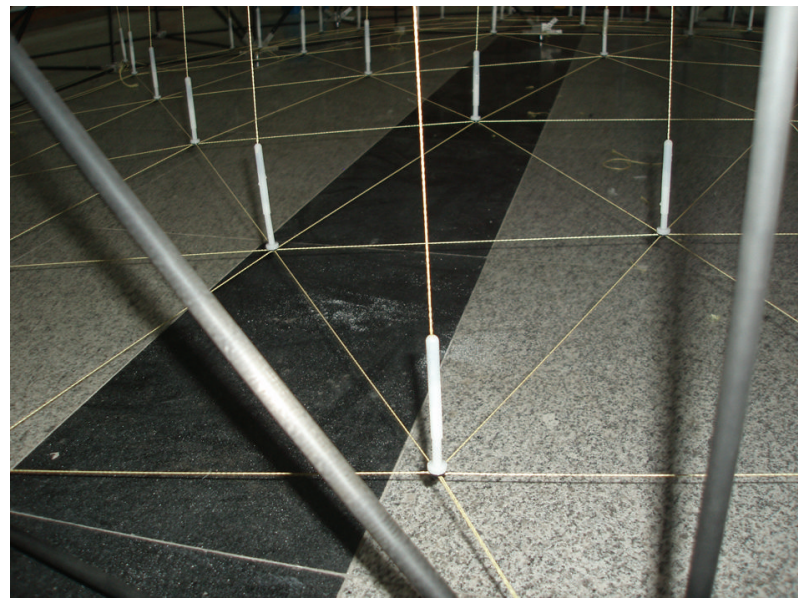

(a)

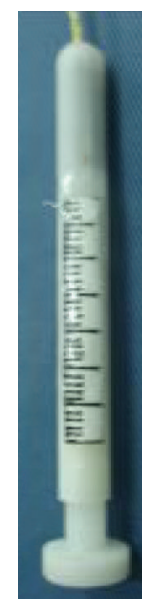

(b)

Figure 11: Adjustment mechanism of the vertical adjustable cables.

The antenna model is suspended by the suspension cables, and these suspend cables keep in vertical state during the test. The other ends of the suspension cables are connected with the plan orbit and moves along the orbit. To avoid the deformation of the orbit becoming large, the rigid of the support frames and the orbit is required to be high.

Another test facility reducing the effect of the gravity force is shown in Figure 13. Universal hinge joints are assembled at the down seven-strut joints of the mesh antenna. The mesh antenna model is placed on the granite ground. Because the friction coefficient between the joints and the granite ground is assumed to be small, the joints can move in the plane smoothly. This test facility is easily realized and has been used in the deployment test, which is shown in Figure 14. The test result showed that the antenna driven by two motors can be deployed successfully. The power of the motors used for the deployment test is $15.5 \mathrm{w}$. It is mentioned that this test facility cannot reduce the influence of the gravity force to the deployment process completely. The first test facility is manufactured and will be used in the future.
The cable net reflector is a flexible structure and therefore its surface accuracy was best determined using a noncontact measurement approach, to avoid any unintentional local deformation caused by contact forces. To meet this requirement, a photogrammetric measurement system based on the PhotoModeler software packages was selected. There are 61 targets fixed in the joints of the front cable net surface in the measurement test. Three cameras were needed in the measurement test, as shown in Figure 15. After the measurement and the numerical analysis, the coordinates of each target point were obtained. The test result surface was compared to the design surface and the adjusted values of the vertical adjustable cables were determined. Then the adjustment of each vertical adjustable cable was finished by hand and the reflective surface accuracy was measured again. The measurement after the adjustment was occurred 20 times in this test. The RMS of the reflective surface in each measurement step is shown in Figure 16. From the test results after 20 steps, the final RMS of the reflective surface is $0.238 \mathrm{~mm}$. It is seen that the surface accuracy is high and the 


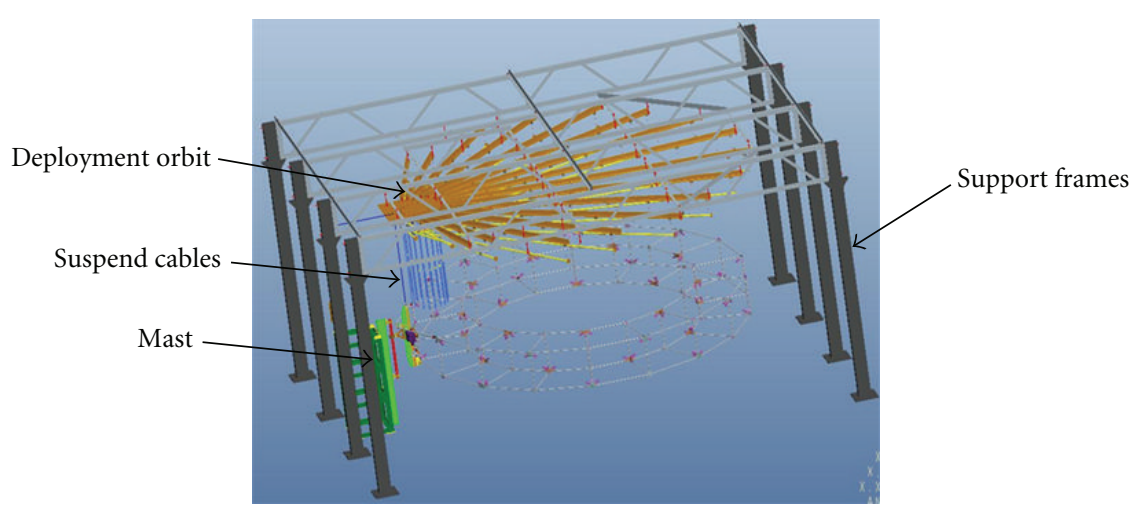

FIGURE 12: Suspension system for the deployment test.

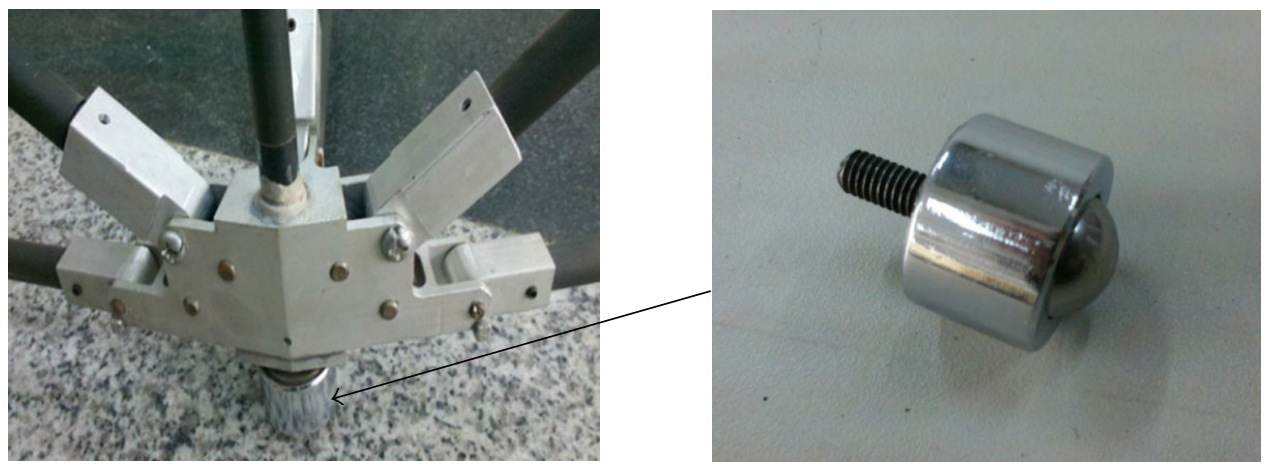

Figure 13: Universal hinge joint system.

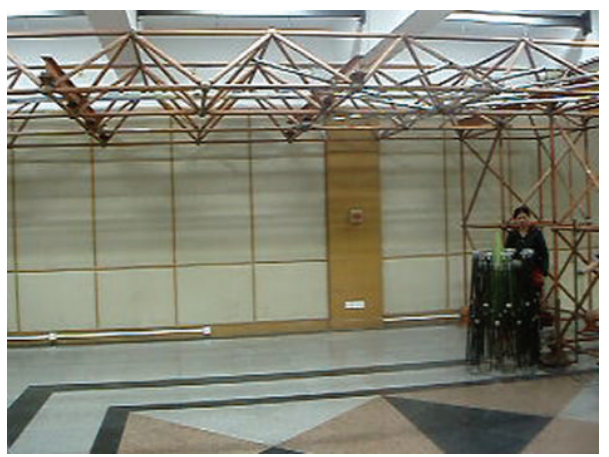

(a)

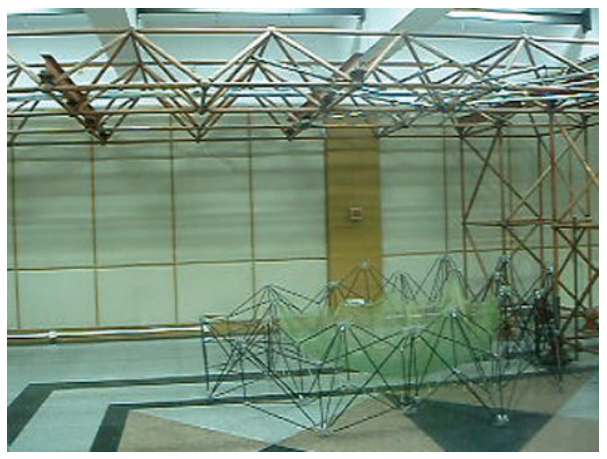

(c)

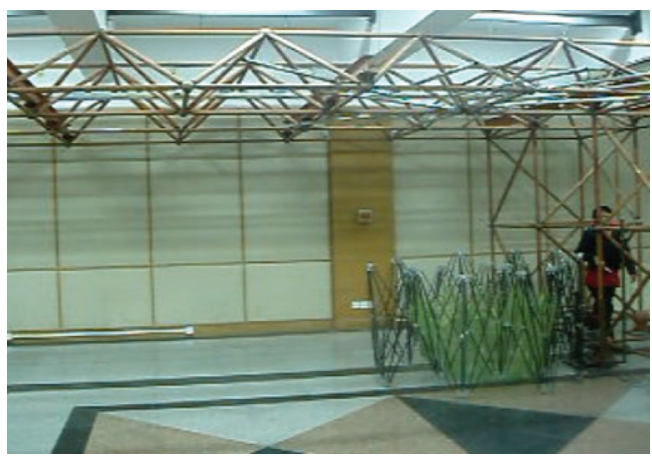

(b)

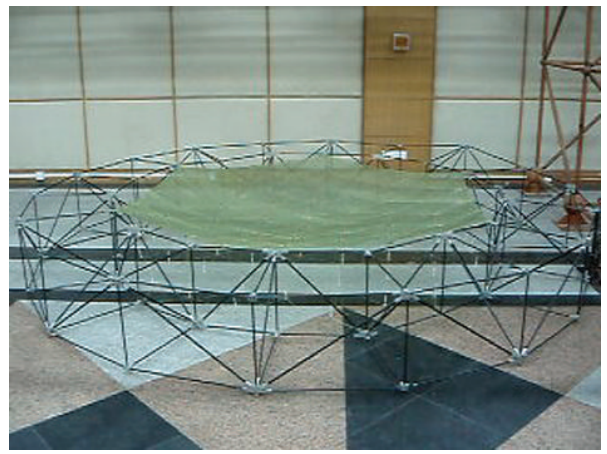

(d)

FIGURE 14: Deployment test of the antenna model. 


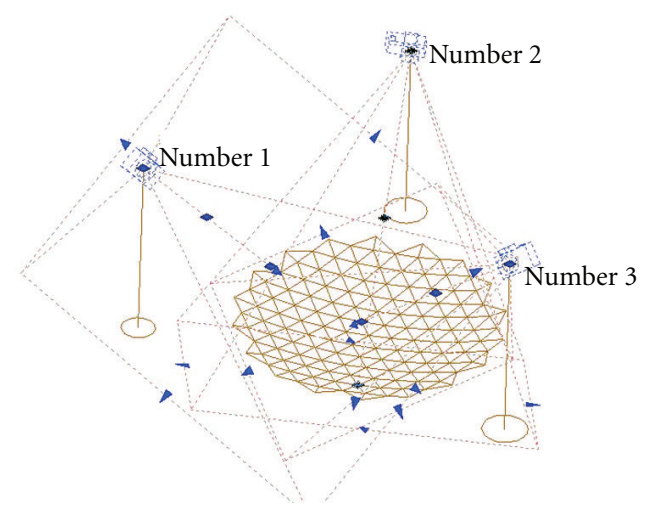

Figure 15: Measurement of the surface accuracy.

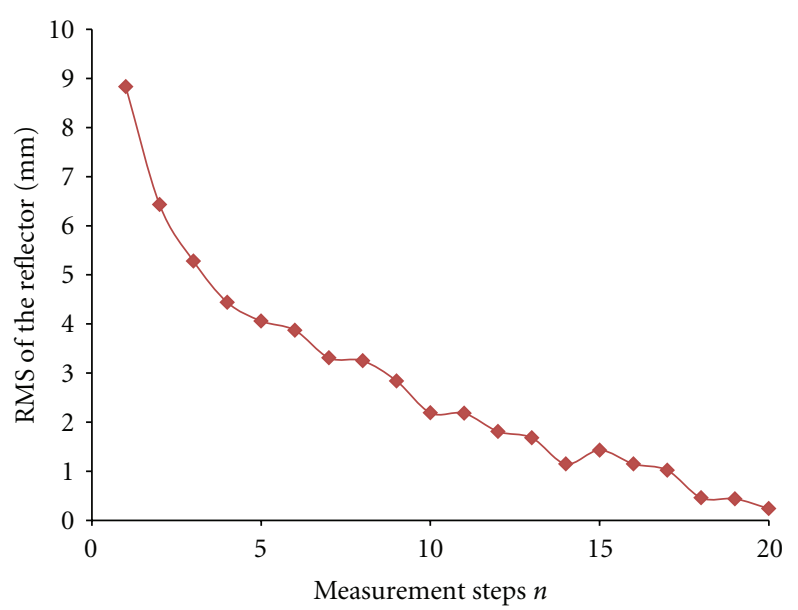

FIgURE 16: RMS in each measurement step.

joints of the front cable net surface are adjusted to the design parabolic surface.

\section{Antenna Patterns Analysis}

The section aims at the antenna pattern analysis of the deployable mesh antenna. The Ticra software Grasp is utilized to analysis the antenna patterns based on the real coordinates obtained from the measurement test. The primary feed horn operates in two frequency bands of 0.5 and 1.0 GHz. The antenna patterns based on the configuration after measurement step 10 are showed in Figure 17. In these figures, the black line represents $E$ plane and the red line represents $H$ plan. The antenna patterns based on the final configuration of the reflective surface are showed in Figure 18. In these figures, the black line represents $E$ plane and the blue line represents $H$ plan. It can be seen that the antenna patterns are prefect in the low-frequency range (less than $1.0 \mathrm{GHz}$ ). Compared with the RMS of the reflective surface, the adjustment does not correspond to a significant increase of electrical performances.

When the frequency band is higher $(3.0 \mathrm{GHz}$ e.g.), the aberrance of the antenna pattern will be very bad and unacceptable, as shown in Figure 19. The reason of this is the reflective surface of this type of mesh antenna consists of a series of triangle grids. The size of triangle grids in the demonstration model is a little large, and it is not suitable for high frequency range. This can be explained by the Ruze's rule [14]. The final RMS $(0.238 \mathrm{~mm})$ is about $0.4 \%$ wavelength and $0.8 \%$ wavelength for $0.5 \mathrm{GHz}$ and $1 \mathrm{GHz}$, respectively, while it is $2.38 \%$ wavelength for $3 \mathrm{GHz}$; it is much bigger than the $1 \%$ wavelength criteria by Ruze's rule.

\section{Conclusion}

A type of deployable mesh antenna consists of the doublering deployable trusses, and the cable net reflector was proposed and investigated. The structural design concept of the deployable mesh antennas was presented. The mechanical measurement tests of the antennas on the ground including test facilities, deployable test, and measurement and adjustment test were introduced. The mass of the entire model is $15.1 \mathrm{Kg}$. The height of the folded configuration is $1.23 \mathrm{~m}$ and the diameter of that is $0.52 \mathrm{~m}$. The test results showed that the antenna driven by two motors can be deployed successfully, and the surface accuracy is high after adjustment and measurement. The antenna pattern was analyzed based on the real surfaces of the reflector obtained 


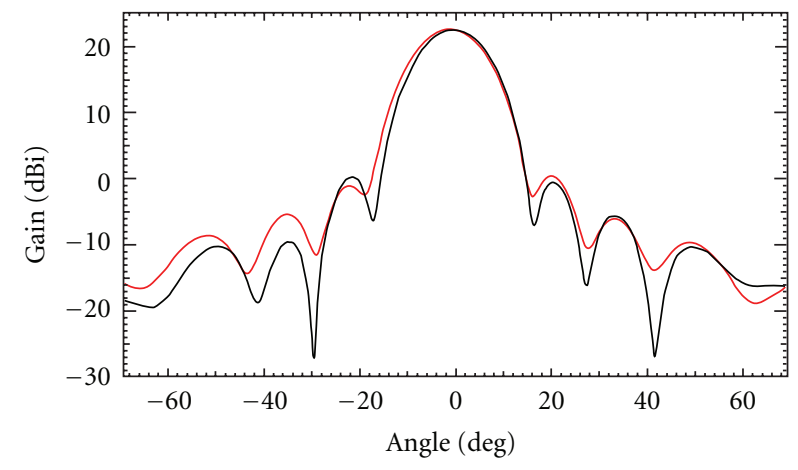

(a) $0.5 \mathrm{GHz}$

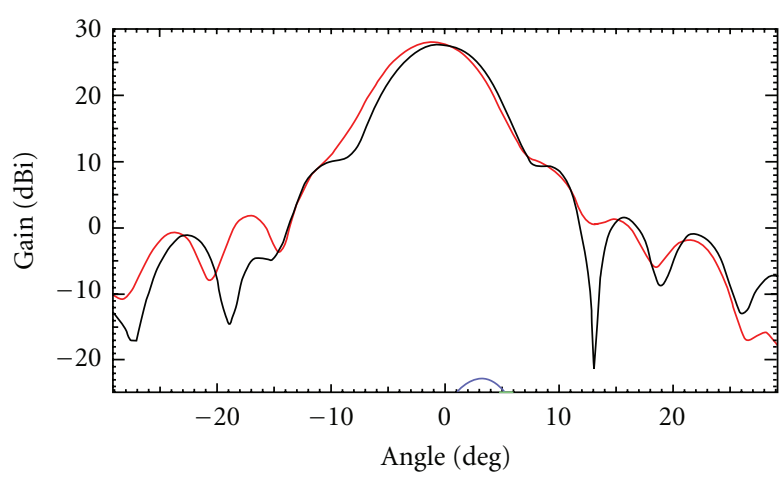

(b) $1.0 \mathrm{GHz}$

Figure 17: Antenna patterns of the surface from measurement step 10.

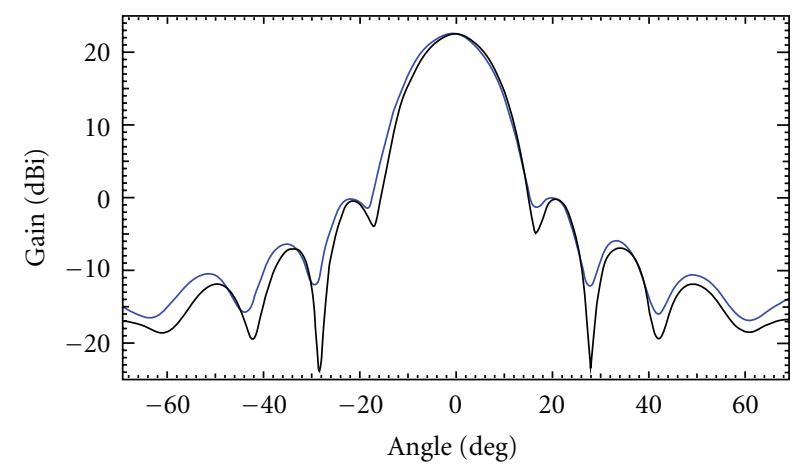

(a) $0.5 \mathrm{GHz}$

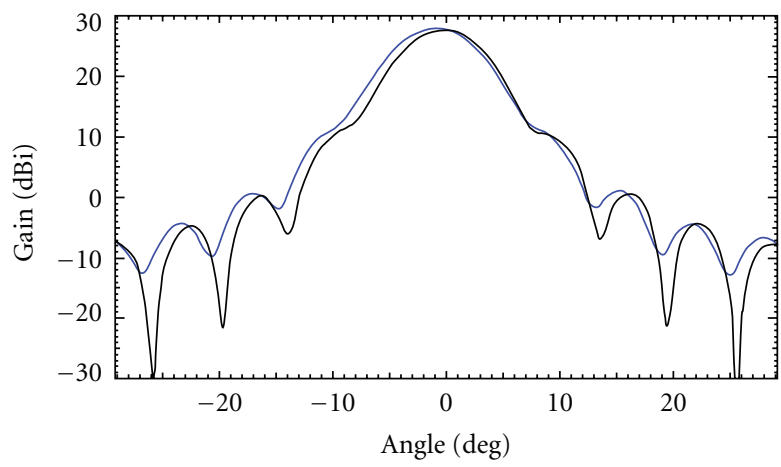

(b) $1.0 \mathrm{GHz}$

Figure 18: Antenna patterns of the final reflective surface.

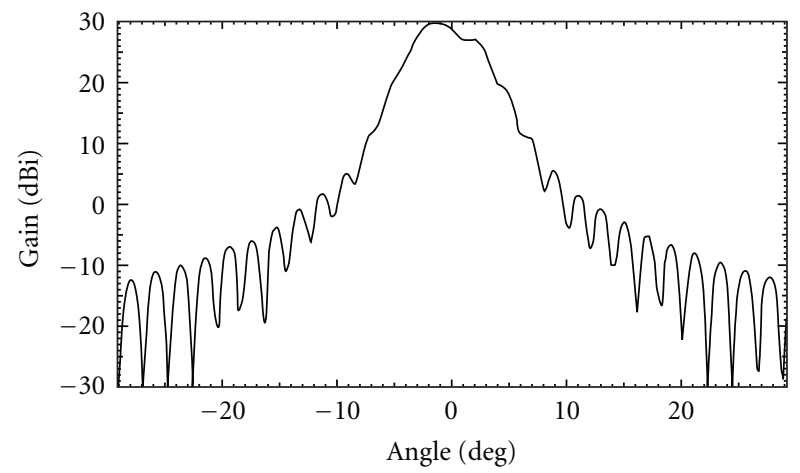

FIgURE 19: Antenna pattern of the final surface $(3.0 \mathrm{GHz})$.

by the reflective surface accuracy measurement. The analytic results indicated that this type of mesh antenna is suitable for low-frequency communication. The reason of the bad antenna pattern for the mesh antenna was also discussed.

To apply this type of mesh antenna in high-frequency communication, the mesh number of the radial cable needs to be large and the size of triangle grids in the reflective surface is designed to be small. But this increases the number of the vertical adjustable cables, the adjustment after installation would be difficult, and the weight and the driven force for deployment would be increased as well. Therefore, the optimize design of the structure-electromagnetic performance for this type of deployable mesh antenna is interesting in the future.

\section{Acknowledgments}

This work was supported by grants from National Natural Science Foundation of China (Grant no. 51008271) and the Fundamental Research Funds for the Central Universities (2012FZA4026).

\section{References}

[1] M. W. Thomson, Astromesh deployable reflectors for Ku- and Ka-band commercial satellites, AIAA-2002-2032.

[2] M. W. Thomson, “The Astromesh deployable reflector," IEEE Antennas and Propagation Society, vol. 3, pp. 1516-1519, 1999.

[3] S. Morterolle, B. Maurin, J. Quirant, and C. Dupuy, "Numerical form-finding of geotensoid tension truss for mesh reflector," Acta Astronautica, vol. 76, pp. 154-163, 2012.

[4] Z. W. Wang, T. J. Li, and Y. Y. Cao, "Active shape adjustment of cable net structures with PZT actuators," Aerospace Science and Technology. In press.

[5] T. H. Lee, R. C. Rudduck, and M. C. Bailey, "Surface distortion analysis applied to the hoop/column deployable mesh reflector 
antenna," IEEE Transactions on Antennas and Propagation, vol. 37, pp. 452-458, 1992.

[6] F. Zheng, M. Chen, and S. Zhu, "Structure-electromagnetic analyses of large deployable mesh antenna for space application," in Asia International Symposium on Mechatronics, pp. 529-533, Hokkaido University, Hokkaido, Japan, August 2008.

[7] A. G. Cherniavsky, V. I. Gulyayev, V. V. Gaidaichuk, and A. I. Fedoseev, "Large deployable space antennas based on usage of polygonal pantograph," Journal of Aerospace Engineering, vol. 18, no. 3, pp. 139-145, 2005.

[8] K. A. Takamatsu and J. Onoda, "New deployable truss concepts for large antenna structures or solar concentrators," Journal of Spacecraft and Rockets, vol. 28, no. 3, pp. 330-338, 1991.

[9] H. Tsunoda, K. Hariu, and Y. Kawakami, Structural design and deployment test methods for a large deployable mesh reflector. AIAA-97-1148:2963-2971.

[10] J. Quirant, F. Cevaer, S. Morterolle, B. Maurin, and J. F. Dub, "Conceptual design and analysis of a deployable structure with flexible joints," Journal of Aerospace Engineering, vol. 24, no. 3, pp. 277-284, 2011.

[11] Z. You and S. Pellegrino, "Cable-stiffened pantographic deployable structures part 2: mesh reflector," AIAA Journal, vol. 35, no. 8, pp. 1348-1355, 1997.

[12] B. P. Nagaraj, R. Pandiyan, and A. Ghosal, "A constraint Jacobian based approach for static analysis of pantograph masts," Computers and Structures, vol. 88, no. 1-2, pp. 95-104, 2010.

[13] B. P. Nagaraj, R. Pandiyan, and A. Ghosal, "Kinematics of pantograph masts," Mechanism and Machine Theory, vol. 44, no. 4, pp. 822-834, 2009.

[14] J. Ruze, "Antenna tolerance theory," IEEE Proceedings, vol. 54, pp. 633-640, 1966. 

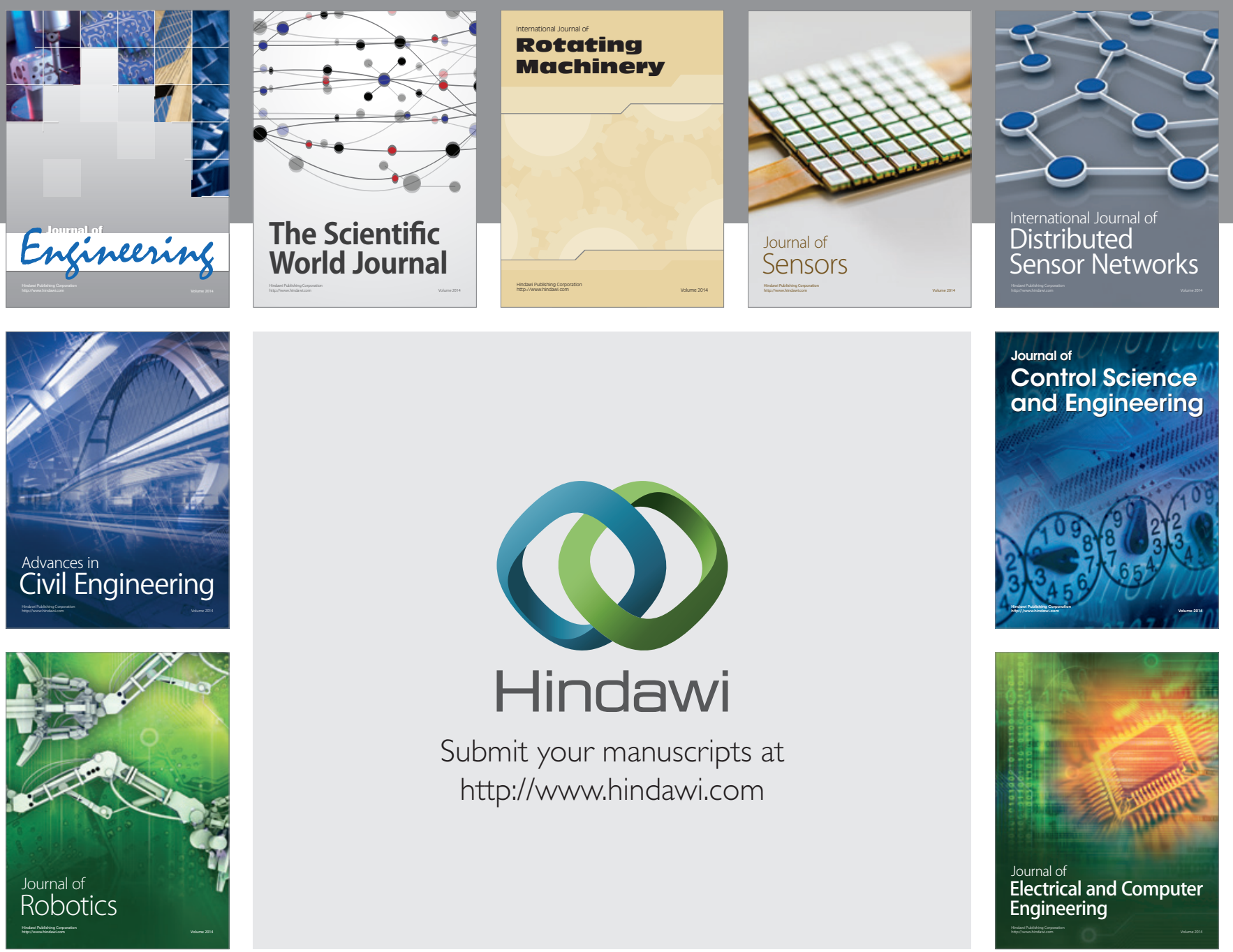

Submit your manuscripts at

http://www.hindawi.com
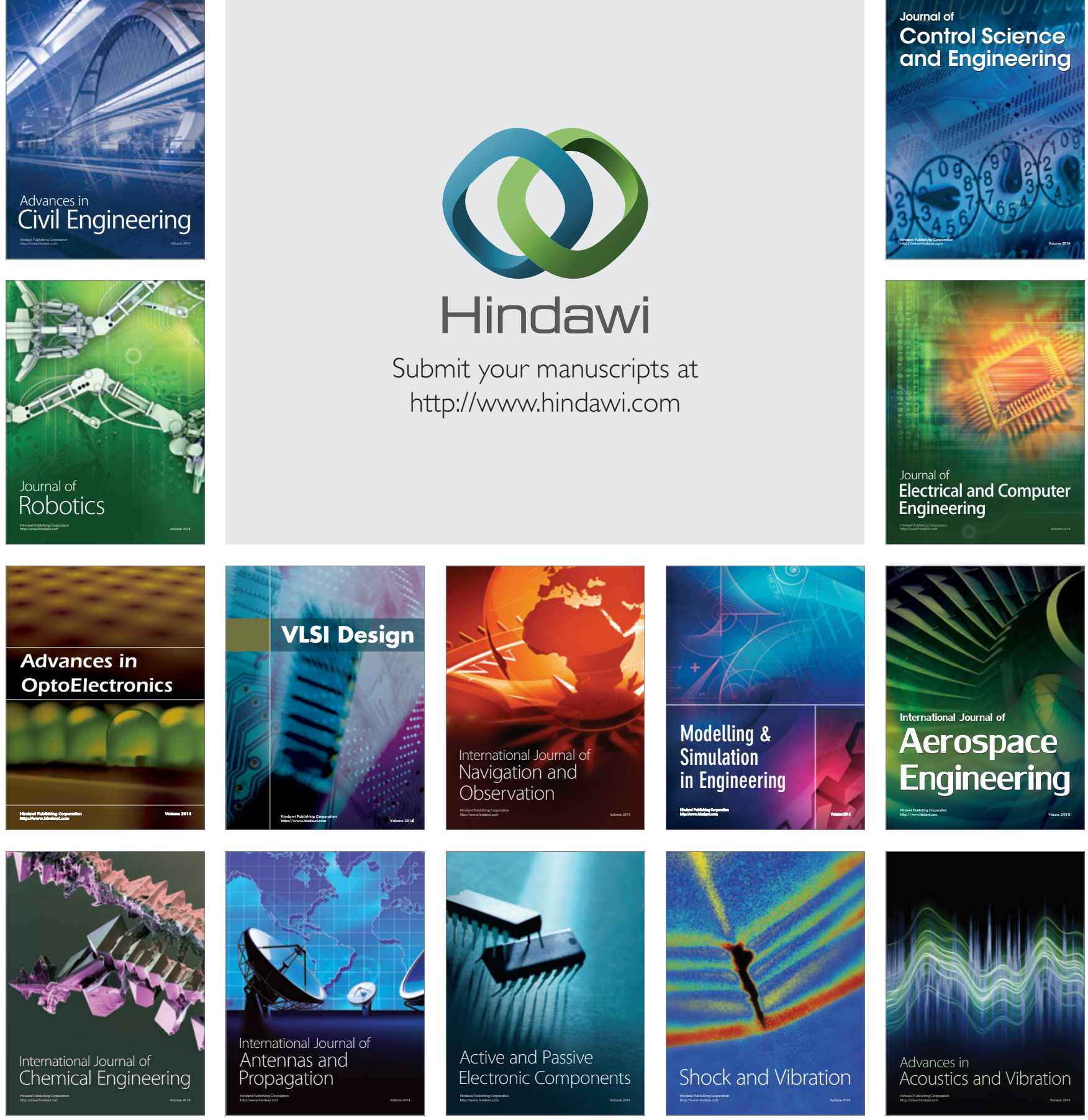